\title{
EXPRESSION OF INTERMEDIATE FILAMENT NESTIN IN BLOOD VESSELS OF NEURAL AND NON-NEURAL TISSUES
}

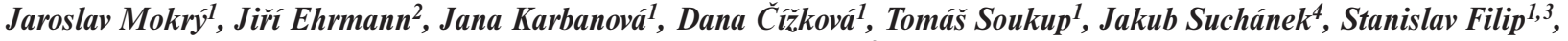 \\ Zdeněk Kolár̆ $\check{r}^{2}$
}

Charles University in Prague, Faculty of Medicine and University Hospital Hradec Králové, Czech Republic: Department of Histology and Embryology ${ }^{1}$, Department of Oncology and Radiotherapy ${ }^{3}$, Department of Dentistry ${ }^{4}$; Palacky University Medical Faculty, Olomouc, Czech Republic: Department of Pathology²

\begin{abstract}
Summary: Our previous findings performed in rat tissues demonstrated that intermediate filament nestin is expressed in endothelial cells of newly formed blood vessels of developing organs and neural transplants. The aim of the present study was to identify other cellular markers expressed in nestin-positive (nestin ${ }^{+}$) blood vessels.

To reach this goal we performed double immunofluorescent study to co-localize nestin with endothelium-specific markers (CD31, CD34 II, vimentin) or markers of perivascular cells (GFAP, SMA) in paraffin-embedded sections of normal human brain tissue, low- and high-grade gliomas, postinfarcted heart and samples of non-neural tumours.

Our findings documented that all the samples examined contained blood vessels with different ratio of nestin ${ }^{+}$endothelial cells. Double immunostaining provided unambiguous evidence that endothelial cells expressed nestin and allowed them to distinguish from other nestin ${ }^{+}$elements (perivascular astrocytic endfeet, undifferentiated tumour cells, smooth muscle cells and pericytes). Nestin ${ }^{+}$endothelium was not confined only to newly formed capillaries but was also observed in blood vessels of larger calibres, frequently in arterioles and venules.

We conclude that nestin represents a reliable vascular marker that is expressed in endothelial cells. Elevation of nestin expression likely corresponds to reorganization of intermediate filament network in the cytoskeleton of endothelial cells in the course of their maturation or adaptation to changes in growing tissues.
\end{abstract}

Key words: Nestin; Endothelium; Human glioma; Regenerating myocardium

\section{Introduction}

Intermediate filament protein nestin is temporarily expressed in undifferentiated cells derived from nervous $(7,9$, $12)$, muscular $(9,10,18)$, and few other tissues $(8,11,14$, 22, 24). Following cell differentiation, nestin expression is downregulated and it is replaced with another type of intermediate filament $(18,19)$.

Our previous findings performed in rat tissues demonstrated that nestin is expressed in endothelial cells of newly formed blood vessels of developing intra- and extraembryonic tissues (14). In the adult rat tissues, nestin is absent in most vascular endothelial cells but appears in blood vessels nourishing growing neural transplants and brain tumours. Based on these findings, we hypothesized that expression of intermediate filament nestin is associated with formation of new endothelial cells. To confirm whether nestin represents a marker of undifferentiated endothelial cells in human tissues, we investigated its presence in human em- bryonic tissues, tumours, regenerating cardiac muscle, and physiologically growing organs like the corpus luteum (13, 16).

Using immunohistochemical detection against a single antigen, we found that nestin was expressed under all these conditions of vascular development. In tissues that consist of nestin-positive elements, like nervous tissues, distinction of positive endothelial cells from other immunoreactive elements is sometimes ambiguous.

For that reason in the present study, we performed double immunostaining of nestin with endothelial markers CD31, CD34 II and vimentin that gave evidence that nestin could be considered as a reliable marker of vascular endothelium. We documented that nestin was expressed not only in newly formed capillaries but also in larger blood vessels. Moreover, we described other vascular and perivascular nestin ${ }^{+}$elements that occurred in human blood vessels of the intact brain tissue, human gliomas, non-neural tissues and regenerating myocardium. 


\section{Material and Methods}

\section{Histology}

Tissue blocks taken from foetal and adult human tissues were immersed in a neutral buffered formalin solution, for five days at room temperature. Tissue blocks were then dehydrated in increasing alcohols and embedded in paraffin. Serial seven-micron-thick coronal sections were cut from paraffin blocks, attached to the slide with poly-L-lysine, and the middle section in each series was stained with haematoxylin-eosin for histological examination.

\section{Immunohistochemistry}

For immunohistochemistry, paraffin-embedded sections were deparaffinized with xylene and rehydrated in decreasing ethanols to water. Sections were incubated for 20 min in methanol containing $1 \%$ hydrogen peroxide to reduce "endogenous peroxidase activity". Because microwave pretreatment enables re-establishment of an original conformation of epitopes modified after fixation, we exposed sections to microwaves in sodium citrate solution for $2 \times 5$ min at 700 watts. After thorough washing in $0.2 \mathrm{M}$ Tris$\mathrm{HCl}$ buffer containing $0.3 \%(\mathrm{v} / \mathrm{v})$ Triton X-100, the sections were exposed to a primary monoclonal anti-human nestin antibody (10C2, 1:200, Chemicon International, Temecula, CA) for $45 \mathrm{~min}$ at room temperature. After washing, sections were incubated with donkey secondary anti-mouse antibody conjugated to horseradish peroxidase or Cy3 fluorochrome (Jackson Immunoresearch Laboratories, West Grove, USA). To perform double immunofluorescence, unoccupied antigen-binding sites in the secondary antibody were saturated by incubation with normal mouse serum followed by incubation in excess of unconjugated monovalent Fab anti-mouse fragment raised in donkey, diluted 1:200 for 45 min (Jackson Immunoresearch Laboratories). The second primary monoclonal antibody was directed against endothelial markers anti-CD31 (JC/70A, 1:200, DakoCytomation, Glostrup, Denmark), anti-CD34 II (QBEnd 10, 1:50, DakoCytomation), vimentin (V9, 1:40, Sigma, Saint Louis, USA), against glial fibrillary acidic protein (GFAP; GA-5, 1:400, Sigma) or smooth muscle actin (SMA; 1A-4, 1:400, Sigma). The binding sites of this antibody were visualized with biotinylated donkey anti-mouse antibody and streptavidin conjugated to Alexa 488. For immunoperoxidase staining, signal was detected with 3,3'-diaminobenzidine tetrahydrochloride and hydrogen peroxide and after dehydration, sections were counterstained with methyl green. For immunofluorescence, sections were counterstained with DAPI and mounted in polyvinylalcohol/glycerol with 1,4-diazobicyclo-[2.2.2]-octane (DAB$\mathrm{CO})$. Serial sections processed with omission of the primary antibody were used as controls. Prior to double immunostaining, we first performed a single detection for each marker in serial sections and after each method confirmed presence of both antigens in the tissue, the other serial sections were processed for double immunostaining.
Sections were examined in Olympus BX-51 microscope equipped with epifluorescence and DP-70 digital camera.

\section{Results}

In the intact adult human brain, most vascular endothelial cells did not express nestin. A weak signal for nestin was detected in some astroglial cell processes. Identity of these thin astrocytic processes was confirmed with antiGFAP immunohistochemistry. Frequently these processes were apposed to vascular surfaces forming the perivascular limiting membrane (Fig. 1A). In larger blood vessels, where the perivascular limiting membrane was separated from endothelial cells by a layer of the adventitial connective tissue, it was rarely possible to distinguish a single nestin-positive $\left(\right.$ nestin $\left.^{+}\right)$endothelial cell. Occasionally nestin ${ }^{+}$vascular endothelial cells were observed also in arterioles. But generally most brain blood vessels were immunonegative for nestin. There were only few immunopositive endothelial cells per section.

Nestin $^{+}$capillaries were described in variety of brain tumours. In this study, we chose specimens of low- and highgrade astrocytomas to observe nestin-immunoreactivity in blood vessels. The solid tumours contained capillaries and larger blood vessels that revealed nestin-immunopositivity. Nestin was also recognized in blood vessels in the vicinity to tumour. Although protein nestin was expressed in most endothelial cells of the tumour capillary network, their visualisation with anti-nestin immunohistochemistry was problematic due to the presence of nestin ${ }^{+}$tumour cells and nestin $^{+}$reactive astrocytes that were found in a close relationship to capillaries (Figs. 1B,C). Tiny nestin ${ }^{+}$capillaries were well visible in those parts of paraffin-embedded sections where tumour cells did not express nestin. In the other sites, nestin ${ }^{+}$endothelia were identified with the help of simultaneous detection of another endothelial marker, CD31, CD34 II or vimentin. Mostly, these markers were co-expressed by the same cells. Inside the tumours, nestin was not expressed exclusively in capillaries but it was also identified in most endothelial cells lining lumina of arterioles (Fig. 1B). The cytoplasm of these epithelial cells was intensely stained including slim elongated processes and perinuclear region; no staining was seen in cell nuclei. Smooth muscle cells in the tunica media were usually devoid of staining; few positive cells appeared in the tunica adventitia. In large veins, nestin ${ }^{+}$endothelia were also present. However, distribution of nestin ${ }^{+}$endothelial cells was not homogenous. Sometimes they were localized in some areas of luminal surfaces whereas in the adjacent area, only few positive endothelial cells were mixed with negative cells. Smooth muscle cells situated in the blood vessel wall beyond the endothelial lining were usually nestin- (Figs. 1D,E). Thus in astroglial tumours, nestin ${ }^{+}$endothelial cells were found in blood vessels of different calibres.

Endothelial cells lining blood vessels of intact adult non-neural tissues are relatively stable and express nestin 

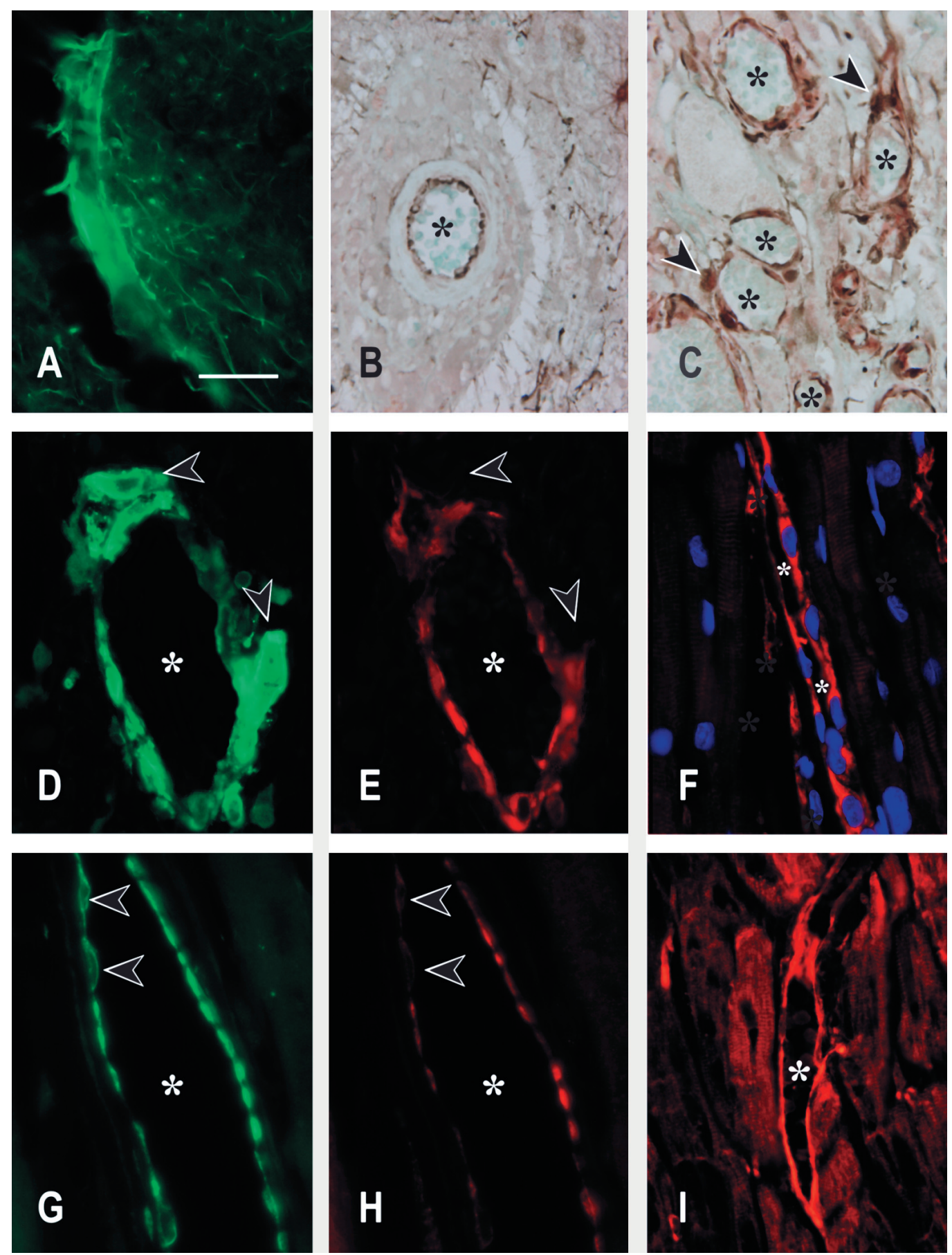

Fig. 1. Visualization of blood vessels using immunohistochemical staining. GFAP expression in perivascular astrocytic end-feet reveals capillaries in the adult human brain (A). In human astrogliomas, nestin is expressed in endothelial lining of blood vessels of different sizes: arterioles (B), venules and capillaries (C). Lumina of blood vessels are indicated by asterisks. In the perivascular space, arrows indicate nestin-positive tumour cells. Other nestin-immunoreactive cells are represented by reactive astrocytes. In venules inside tumours, nestin was expressed in endothelium (E), whereas smooth muscle (arrows) and periendothelial cells expressed SMA (D). In the regenerating heart, nestin was expressed in endothelium of capillaries (F), venules $(\mathrm{H}, \mathrm{I})$ as well as arterioles. Endothelial cells co-expressed vascular markers like vimentin $(G)$. Arrows show perikarya of two vimentin-positive endothelial cells that lack nestin whereas the other endothelial cells in the same venule co-express both markers. Spacebars in A, D, E correspond to $40 \mu \mathrm{m}$, in B, C, to $60 \mu \mathrm{m}$, in F to $50 \mu \mathrm{m}$, in $\mathrm{G}, \mathrm{H}$ to $25 \mu \mathrm{m}$, in I to $70 \mu \mathrm{m}$. 

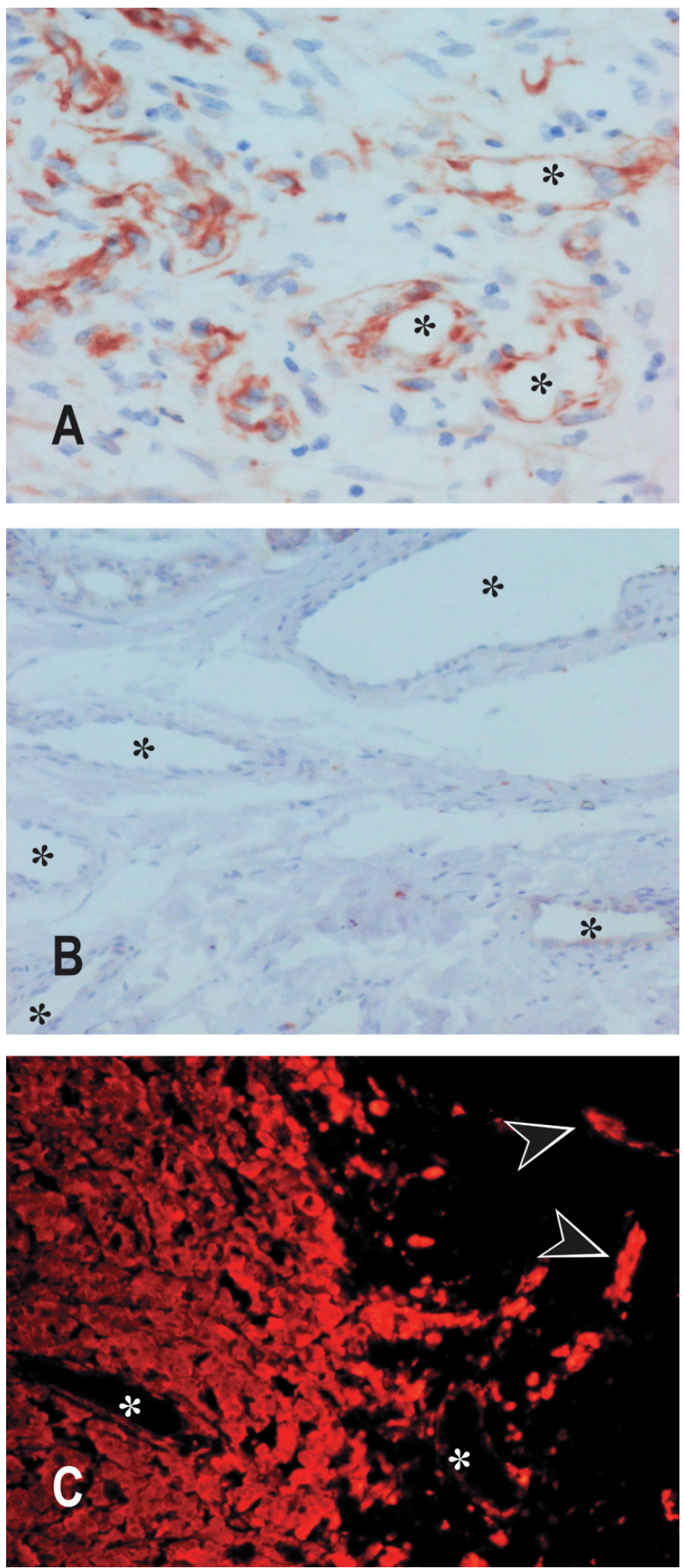

Fig. 2. Immunohistochemical detection of nestin in non-neural tumours. A) Nestin expression in capillary haemangiomas is confined to endothelial cells and pericytes. B) In cavernous haemangiomas, nestin is not expressed in endothelial cells. C) In the malignant melanoma, nestin is expressed both in tumour cells and blood vessels. Arrows indicate nestin-positive capillaries found in the vicinity to tumour. Lumina of blood vessels are indicated by asterisks. 
only sporadically. For example in the skeletal or cardiac muscle tissues, most blood vessels were nestin-negative and only occasionally, a nestin ${ }^{+}$endothelial cell was found to be inserted between other nestin endothelial cells. However, situation changed if the tissue was subjected to an injury that induced tissue regeneration associated with formation of new blood vessels. In the myocardium obtained from necropsy of patients who died 6-10 days postinfarction, ne$\operatorname{stin}^{+}$capillaries were localized to the border zone in the vicinity to the necrotic tissue. While a central part of the necrosis did not contain capillaries, nestin ${ }^{+}$capillaries appeared in the border zone and some grew in the necrotic tissue. In these growing capillaries, all endothelial cells contained nestin in their cytoplasm (Figs. 1F,I). At tips of such capillaries, budding endothelial cells sent tiny irregular cytoplasmic processes. Observation of capillaries in longitudinal sections allowed to distinguish that level of nestin protein slightly differed between neighbouring endothelial cells. In slightly distant areas of the myocardium adjacent to the necrotic tissue, nestin-positivity was not confined only to capillaries but was also identified in the endothelium of arterioles and venules. Although nestin-immunoreactivity was confined to the cytoplasm of endothelial cells, some other nestin ${ }^{+}$elements were recognized in the cardiac muscle tissue. These included smooth muscle cells, pericytes, some interstitial cells and cardiomyocytes. Cardiac muscle cells were easily distinguished because of their larger size and typical morphology. As interstitial cells we call those elements that were localized in the connective tissue outside the blood vessels. Rare nestin ${ }^{+}$cells were seen in the interstitium; they were usually spindle-shaped with elongated processes. Smooth muscle cells were identified according to their morphology and characteristic position in the tunica media of the arterioles. Although these cells expressed nestin, intensity of immunostaining was faint indicating that nestin did not reach the levels that were typical for endothelial cells. Nestin ${ }^{+}$pericytes were frequently observed near the vascular lumen but they were separated from blood cells by a continuous layer of endothelial cells. Double immunohistochemistry of nestin with endothelial markers confirmed that the most numerous immunoreactive cells were endothelia. All endothelial cells expressed vimentin that was often found to co-localize with nestin although the levels of the latter protein were not uniform. The most intense nestin ${ }^{+}$ endothelium was found in a close contact with nestin ${ }^{+}$cardiomyocytes. Immunohistochemistry against endothelial membranous markers CD31 or CD34 II identified the entire capillary network in the preserved myocardial tissue. Co-expression of nestin with CD31 or CD34 II confirmed that nestin $^{+}$adluminal vascular cells were unambiguously endothelial cells. In the same blood vessel, different endothelial cells expressed different varying levels of nestin. Few endothelia were nestin ${ }^{-}$although the adjacent endothelial cells were nestin ${ }^{+}$(Figs. 1G,H). In areas distant from the infarcted tissue, the ratio between nestin-positive and negative vascular cells was lower than in the regenerating border zone.
In non-neural tumours, we detected nestin in vascular endothelium of vessels nourishing malignant melanomas, renal adenocarcinomas and capillary haemangiomas. In the malignant melanoma of the skin (Fig. 2C), the tumour was formed by nestin ${ }^{+}$neoplastic cells. Nestin ${ }^{+}$blood vessels vascularized the entire tumour but immunoreactive endothelial cells were found in a close contact with nestin ${ }^{+}$ tumour cells. Nestin ${ }^{+}$capillaries were better seen at the boundary between the tumour and the host tissue where they were not accompanied by tumour cells. In haemangiomas, a high level of nestin expression was detected in endothelial cells and pericytes of capillary haemangiomas (Fig. 2A). In cavernous haemangiomas (Fig. 2B), these elements were nestin-negative. In Grawitz adenocarcinomas of the kidney, nestin expression was heterogeneous. Nestin was observed in endothelial cells, pericytes, some tumour cells and in interstitial cells that were in contact with neoplastic buds.

\section{Discussion}

Intermediate filament protein nestin was originally isolated from the embryonic nervous tissue (9) and considered as a marker of neural stem cells $(7,12)$. This marker was also found to be expressed in other immature neural cells and also in a variety of non-neural tissues like myoblasts $(9,10,18)$, odontoblasts $(22)$, Sertoli cells (8) etc. Nestin expression observed in cerebral vessels was first erroneously considered for non-specific staining caused by red blood cells observed in immunoperoxidase histochemistry. Nestin was preferably and frequently used as a marker of undifferentiated cells in neurobiology. For that reason nestin-immunoreactivity was early recognized in endothelial cells of blood vessels nourishing the brain tumours $(3,6,23)$. However, a close apposition of other nestin $^{+}$elements to endothelial cells prevented to distinguish well distinct elements expressing the same protein (endothelium from astroglial and tumour cells). In our previous experimental work (15), we used Rat-401 antibody that is specific to rat and mouse nestin to identify expression of this intermediate filament in the rat developing brain. In the adult brain tissue, we observed nestin ${ }^{+}$vasculature in adult rats transplanted intracerebrally with C6 glioma cells or embryonic nervous tissue. While in the $\mathrm{C} 6$ glioma, nestin ${ }^{+}$capillaries were accompanied with nestin ${ }^{+}$ tumour cells, in the neural grafts 3 weeks posttransplantation, nestin levels ceased in differentiated neural elements but remained in endothelial cells of capillaries, which helped to visualise the entire newly formed capillary network inside the transplant. We looked at developing non-neural rat tissues and found abundant immunoreactivity in blood vessels of all growing organs as well as extraembryonic rat tissues (14). All these data confirmed that nestin represented a novel marker that was expressed by newly formed endothelial cells. In our recent study performed with anti-human nestin-specific antibody, we gave evidence that nestin was 
expressed in endothelium of blood vessels in a variety of developing human organs (13). In intact adult tissues, nestin disappeared from the vascular network. Therefore we focused on processes associated with neovascularization in adult tissues like tumourigenesis, regeneration and cyclic changes in tissues of female reproductive tract that occurred in response to cyclic hormonal regulation. We proved that all these processes including cardiac muscle regeneration and formation of the corpus luteum were associated with nestin expression in endothelial cells $(13,16)$. Similar findings were made by Aihara et al. (1) who described nestin-immunoreactivity in neoangiogenic vessels in the uterine endometrium.

In the present study, we performed double immunofluorescent staining to identify phenotypes of vascular nestin ${ }^{+}$ cells in human intact neural and non-neural tissues as well as in neural and non-neural neoplasias. Nestin-immunoreactive elements associated with blood vessels may include endothelial cells, smooth muscle cells, pericytes, perivascular astroglia (in capillaries with the blood-brain barrier) and perivascular tumour cells. Smooth muscle cells may express low levels of nestin. Their immunoreactivity is lower than that of the lining epithelial cells, myocytes express SMA and they are usually well recognized morphologically. Pericytes represent the other candidates of nestin ${ }^{+}$vascular cells. As pericytes are considered those vascular elements that do not line the lumen but they are in contact with endothelial cells. Most authors identify pericytes according to expression of SMA, which is however characteristic for resting pericytes only. In activated pericytes involved in the process of angiogenesis and in formation of angiogenic phenotypes as do endothelial cells, SMA is not expressed (25). Such pericytes are likely nestin ${ }^{+}(2)$ but their precise identification was beyond limits of techniques used in this study. Perivascular astrocytes contacting cerebral blood vessels with their end-feet were identified on the basis of GFAP expression and characteristic morphology. Nestin ${ }^{+}$ tumour cells that migrated through the adventitial connective tissue of blood vessels were distinguished from other nestin $^{+}$vascular elements if they exhibited different morphological features, e.g. voluminous cytoplasm as shown in Fig. 1C. Nestin ${ }^{+}$endothelial cells were identified according to their typical morphology: they lined a vascular lumen as a single layer of flattened or elongated cells. Because tiny cytoplasmic endothelial extensions could be overlooked at light microscopy level we performed simultaneous immunostaining that allowed to co-localize nestin with markers of perivascular cells (GFAP or SMA) or endothelial cells (CD31, CD34 II or vimentin). This observation confirmed that nestin ${ }^{+}$cells lining the vascular lumina are really endothelial cells. In most blood vessels that contained nestin ${ }^{+}$ endothelial cells, the immunostaining with CD31 and CD34 II was almost identical revealing only minor variances. Detection of another intermediate filament protein, vimentin, showed that adjacent endothelial cells could express different levels of nestin and vimentin. This finding may indicate that some endothelial cells reached more advanced differentiation (and expressed more vimentin) than other less differentiated endothelial cells (that still contained high levels of nestin).

The intermediate filament nestin plays an important role in building up the cytoskeleton of fully differentiated endothelial cells that have to withstand large mechanical strains to which tiny cytoplasmic processes are subjected in blood vessels. Sugawara et al. (21) described that proliferating endothelial cells in static cultures expressed a high level of nestin. But nestin expression was down regulated after a 12-hour-exposition to a physiological shear stress flow that stopped proliferation and induced the endothelial cell differentiation. Nestin cannot form filamentous network in the cytoplasm but co-polymerizes with vimentin (20). Less stable nestin-vimentin heteropolymers promote disassembly and restructuring intermediate filament network in dividing and migrating cells (5). In postmitotic cells, unstable heteropolymers are replaced with the definitive intermediate filament vimentin which forms stable and more rigid oligomers. Our observation, that in the same blood vessel, few endothelia were vimentin ${ }^{+} /$nestin $^{-}$although the adjacent endothelial cells were vimentin ${ }^{+} /$nestin $^{+}$, may be interpreted as gradual differentiation of endothelial cells. Fully differentiated cells had lost nestin whereas immature cells still exhibited varying levels of this intermediate filament protein.

Expression of nestin in proliferating endothelial cells was well documented. In experiments when pregnant rats were injected with thymidine analogue bromodeoxyuridine, we observed nestin in the cytoplasm of endothelial cells that contained this proliferative marker in their nuclei (14). Reorganisation of nestin and changes in its cytoplasmic levels in mitotic and postmitotic cultured cells were published by Sahlgren et al. (17). Aihara et al. (1) described that nestin expression was specific to proliferative endothelium of haemangioblastomas expressing proliferative markers $\mathrm{Ki}-67$ and PCNA (proliferating cell nuclear antigen). They found that nestin expression in endothelium was driven by the first intron of the nestin gene. By examination of nestin coexpression with PCNA in postinfarction cardiac muscle, we detected co-localization of both markers in endothelium of regenerating capillaries (13,16). Recently Amoh et al. (4) used nestin as marker of angiogenic endothelial cells and created an elegant animal model for the visualization of tumour angiogenesis which was based on transplantation of tumour cells in nude transgenic mice with nestin regulatory element-driven green fluorescent protein.

In the present study, we described that nestin in endothelial cells was not confined only to newly formed capillaries but was quite frequently expressed in the lining epithelium of larger blood vessels like arterioles and venules. We observed this phenomenon in blood vessels of brain tumours, in the postinfarction myocardium as well as in the corpus luteum. It is unlikely that in such large blood vessels all endothelial cells are just generated. However further re- 
search in this field is required for elucidation. We may hypothesize that endothelial nestin expression in large blood vessels correspond to reorganization of their cytoskeleton that is associated with adaptation to dynamic changes in the vascular network of growing or regenerating tissues. Nestin expression in endothelial cells can be also related to origin of particular endothelial cells since endothelial cells can be generated by a broad spectrum of cells including primitive haemangioblasts, mesenchymal stem cells, endothelial cells, pericytes, circulating mononuclear cells and different tissue specific stem cells like cardiac stem cells (5) and neural stem cells (26). Different origins of endothelial cells have to involve different differentiation pathways which may differ in time required for intermediate filament network reorganization.

\section{Conclusions}

This work gives unambiguous evidence that vascular endothelial cells express nestin. Moreover it describes other nestin-immunoreactive vascular and perivascular cells. Our findings confirm that nestin is not confined only to angiogenic capillaries but it is expressed also in endothelial cells lining blood vessels of larger calibres.

\section{Acknowledgements}

The authors are thankful to Mrs. Hana Hollerová for her skillful technical assistance. This work was supported by the grant No. MSM0021620820.

\section{References}

1. Aihara M, Sugawara K, Torii S et al. Angiogenic endothelium-specific nestin expression is enhanced by the first intron of the nestin gene. Lab Invest 2004; 84:1581-92.

2. Alliot F, Rutin J, Leenen PJM, Pessac B. Pericytes and periendothelial cells of brain parenchyma vessels co-express aminopeptidase $\mathrm{N}$, aminopeptidase $\mathrm{A}$, and nestin. J Neurosci Res 1999;58:367-78.

3. Almqvist PM, Mah R, Lendahl U, Jacobsson B, Hendson G. Immunohistochemical detection of nestin in pediatric brain tumors. J Histochem Cytochem 2002; 50:147-58.

4. Amoh Y, Yang M, Li L et al. Nestin-linked green fluorescent protein transgenic nude mouse for imaging human tumor angiogenesis. Cancer Res 2005 65:5352-7.

5. Beltrami AP, Barlucchi L, Torella D et al. Adult cardiac stem cells are multipotent and support myocardium regeneration. Cell 2003;114:763-76.
5. Chou YH, Khuon S, Herrmann H, Goldman RD. Nestin promotes the phosphorylation-dependent disassembly of vimentin intermediate filaments during mitosis. Mol Biol Cell 2003;14:1468-78

6. Dahlstrand J, Collins VP, Lendahl U. Expression of the class VI intermediate filament nestin in human central nervous system tumors. Cancer Res 1992;52: 5334-41.

7. Frederiksen K, McKay RDG. Proliferation and differentiation of rat neuroepithelial precursor cells in vivo. J Neurosci 1988;8:1144-51.

8. Frojdman K, Pellieniemi LJ, Lendahl U, Virtanen I, Eriksson JE. The intermediate filament protein nestin occurs transiently in differentiating testis of rat and mouse. Differentiation 1997;61:243-9.

9. Hockfield S, McKay RDG. Identification of major cell classes in the developing mammalian nervous system. J Neurosci 1985;5:3310-28.

10. Kachinsky AM, Dominov JA, Miller JB. Myogenesis and the intermediate filament protein, nestin. Dev Biol 1994;165:216-28.

11. Koláŕ Z, Ehrmann J, Turashvili G, Bouchal J, Mokrý J. A novel myoepithelial/progenitor cell marker in the breast? Virchows Arch 2007;450:607-9.

12. Lendahl U, Zimmerman LB, McKay RDG. CNS stem cells express a new class of intermediate filament protein. Cell 1990;60:585-95.

13. Mokrý J, Čižková D, Filip S et al. Nestin expression by newly formed human blood vessels. Stem Cells Dev 2004; 13:658-64.

14. Mokrý J, Němeček S. Angiogenesis of extra- and intraembryonic blood vessels is associated with expression of nestin in endothelial cells. Folia Biol (Prague)1998; 44:155-61

15. Mokrý J, Němeček S. Cerebral angiogenesis shows nestin expression in endothelial cells. Gen Physiol Biophys 1999;18:25-9.

16. Mokrý J, Pudil R, Ehrmann J et al. Re-expression of nestin in the myocardium of postinfarcted patients. Virchows Arch 2008; DOI 10.1007/s00428-008-0631-8.

17. Sahlgren CM, Mikhailov A, Hellman J et al. Mitotic reorganization of the intermediate filament protein nestin involves phosphorylation by cdc2 kinase. J Biol Chem 2001;276:16456-63.

18. Sejersen T, Lendahl U. Transient expression of the intermediate filament nestin during skeletal muscle development. J Cell Sci 1993;106:1291-1300.

19. Sjoberg G, Jiang WQ, Ringertz NR, Lendahl U, Sejersen T. Colocalization of nestin and vimentin/ desmin in skeletal muscle cells demonstrated by threedimensional fluorescence digital imaging microscopy. Exp Cell Res 1994;214: $447-58$.

20. Steinert PM, Chou YH, Prahland V et al. A high molecular weight intermediate filament-associated protein in BHK-21 cells is nestin, a type VI intermediate filament protein. Limited co-assembly in vitro to form heteropolymers with type III vimentin and type IV $\alpha$-internexin. J Biol Chem 1999;274:9881-90.

21. Sugawara K, Kurihara $\mathrm{H}$, Negishi $\mathrm{M}$ et al. Nestin as a marker for proliferative endothelium in gloimas. Lab Invest 2002;82:345-51.

22. Terling C, Rass A, Mitsiadis TA, Fried K, Lendahl U, Wroblewski J. Expression of the intermediate filament nestin during rodent tooth development. Int J Dev Biol 1995;39:947-56

23. Tohyama T, Lee WMY, Rorke LB, Marvin M, McKay RDG, Trojanowski JQ Nestin expression in embryonic human neuroepithelium and in human neuroepithelial tumor cells. Lab Invest 199;66:303-13.

24. Wagner N, Wagner KD, Scholz H, Kirschner KM, Schedl A. Intermediate filament protein nestin is expressed in developing kidney and heart and might be regulated by the Wilms' tumor suppressor Wt1. Am J Physiol Regul Integr Comp Physiol 2006;291:R779-87.

25. Witmer AN, vanBlijswijk BC, vanNoorden CJF, Vrensen GFJM, Schlingemann RO. In vivo angiogenic phenotype of endothelial cells and pericytes induced by vascular endothelial growth factor-A. J Histochem Cytochem 2004;52:39-52.

26. Wurmser AE, Nakashima K, Summers RG et al. Cell fusion-independent differentiation of neural stem cells to the endothelial lineage. Nature 2004;430: $350-56$.

Submitted June 2008. Accepted September 2008.

\section{Corresponding author:}

Prof. MUDr. Jaroslav Mokrý, Ph.D., Charles University in Prague, Faculty of Medicine in Hradec Králové, Šimkova 870, 50038 Hradec Králové, Czech Republic, e-mail: mokry@lfhk.cuni.cz 